\title{
Reliable estimates in the anisotropic heat conduction problems
}

\author{
Nikolay Banichuk ${ }^{1,2}$, Svetlana Ivanova ${ }^{1}$, Pekka Neittaamäki ${ }^{2}$ and Tero Tuovinen²*
}

\author{
${ }^{*}$ Correspondence: \\ tero.t.tuovinen@jyu.fi \\ ${ }^{2}$ Department of Mathematical \\ Information Technology, University \\ of Jyväskylä, Mattilanniemi 2, Agora, \\ 40014 Jyväskylä, Finland \\ Full list of author information is \\ available at the end of the article
}

\begin{abstract}
The heat conduction problems for anisotropic bodies are studied taking into account the uncertainties in the material orientation. The best estimations of the upper and lower bounds of the considered energy dissipation functional are based on developing new approach consisting in solution of some optimization problems and finding the extremal internal material structures, which realize minimal and maximal dissipation. The motivation of this study comes from paper making processes, and more precisely, drying process, which consumes about $50 \%$ of the energy fed into the paper machine. The understanding of the effect of uncertainties in the process arises from structural properties of paper will provide the possibility to optimize the drying system.
\end{abstract}

Keywords: Uncertainties; Reliable estimates; Heat conduction; Anisotropic material

\section{Introduction}

The problems of incompleteness of data and uncertainties are typical for anisotropic solids and structures having chaotic orientation of small material particles such as grains, crystal or short nanofibers. Different possible compositions of elementary particles with various orientations result in different values of such integral characteristics as a total dissipation energy in the heat conduction problems, total potential energy in the thermoelasticity and thermoconductivity problems. Taking into account the conditions of uncertainties concerning the material orientations it is very important to obtain various estimations of the considered functionals and in particular limiting estimates known as double-sided or bilateral estimates (see book by Banichuk and Neittaanmäki [1]).

The motivation of this study arises from understanding of paper making processes. As is well known that paper product have an anisotropic fibrous structure which properties depend on the making process and its parameters (velocity, tension, etc.). The understanding of heat conduction behaviour in anisotropic material is very critical for optimization of the system. During paper making, the drying process consumes about $50 \%$ of the energy fed into the paper machine; it is the single largest consumer of energy in the paper manufacturing process.

To model the drying of a moving paper web, several models exist in the literature (see e.g. Karlsson [2], Lampinen and Toivonen [3] and Lu and Shen [4]). For a thorough engineering-oriented discussion on paper drying, see the book edited by Karlsson [5]. In

(C) 2014 Banichuk et al.; licensee Springer. This is an Open Access article distributed under the terms of the Creative Commons Attribution License (http://creativecommons.org/licenses/by/4.0), which permits unrestricted use, distribution, and reproduction in any medium, provided the original work is properly credited. 
our study, we have assumed, that the material is not moving. Moreover, we consider the fundamental mathematical setup of the problem, that the results can be applied widely.

In this article, the problem of estimation of dissipation energy characteristics is considered for anisotropic body constituting of the locally orthotropic material. It is assumed that an orientation of the principle axes of orthotropy is not known beforehand at each point of the body and can be distributed by various ways in different parts of the body including chaotic orientation. The search for double-sided estimates is reduced to the solution of optimization problems and finding the extremal orientations of the orthotropy axes.

\section{Heat conduction problem for bodies from locally orthotropic material}

Let us consider heat conduction problem for solid body occupied the domain $\Omega$ (see Figure 1) with the boundary $\Gamma=\Gamma_{g}+\Gamma_{i}$ where $\Gamma_{g} \cap \Gamma_{i}=0$. The material of the body is anisotropic with respect to the heat conduction process described by the known relations (see e.g. Landau and Lifshitz [6] and Nowacki [7])

$$
\mathbf{q}=D \times \nabla \varphi, \quad \varphi=\theta^{-1}
$$

where $\theta$ is a temperature, $\mathbf{q}$ is a vector of the heat flux and $D$ is a heat conduction tensor of the second rank. In the case of absence of the source of heat in the domain $\Omega$, we will use the boundary conditions, governing equation and the quality functional (dissipation of energy) in the following form

$$
\begin{aligned}
& (\varphi)_{\Gamma_{g}}=\varphi^{0}, \quad(\mathbf{n} \times D \times \nabla \varphi)_{\Gamma_{i}}=0 \\
& \nabla \times(D \times \nabla \varphi)=0 \quad x \in \Omega \\
& J=\int_{\Omega} \nabla \varphi \times D \times \nabla \varphi \mathrm{d} \Omega
\end{aligned}
$$

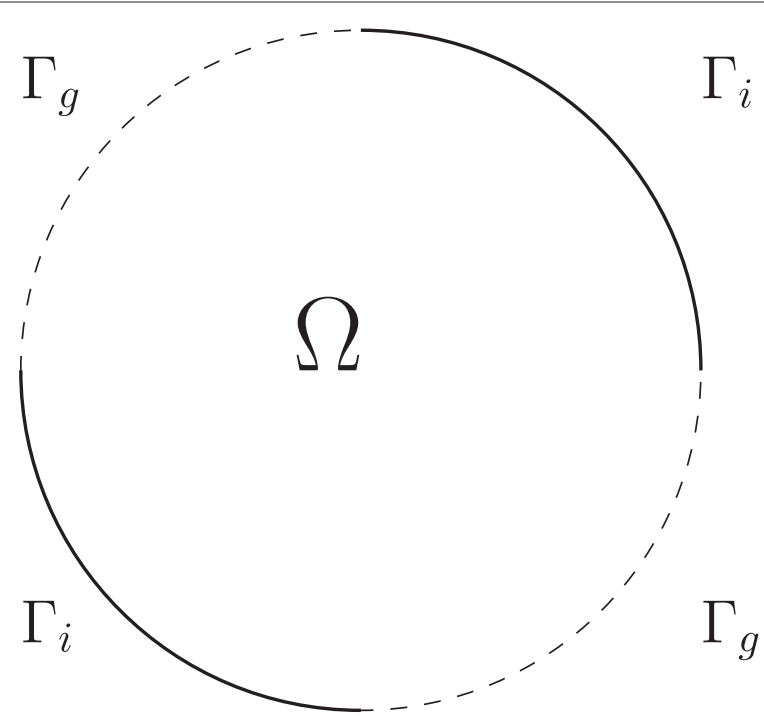

Figure 1 Domain $\Omega$ with given temperature $\Gamma_{i}$ (solid) and thermally insulated $\Gamma_{g}$ (dashed) boundary conditions. 
where $\varphi^{0}$ is a given function specified on $\Gamma_{g}, \mathbf{n}$ is an outwards unit normal vector specified on the part $\Gamma_{i},(\cdot)$ between the vectors means the scalar product and the symbol $\nabla$ is the gradient operator, i.e.

$$
\nabla \varphi=\left\{\frac{\partial \varphi}{\partial x_{1}}, \frac{\partial \varphi}{\partial x_{2}}, \frac{\partial \varphi}{\partial x_{3}}\right\} .
$$

In accordance with the variational principle (see e.g. Berdichevsky [8]), the actual distribution of the function $\varphi$ realizes a minimum for the functional $J$ on the set of admissible functions satisfying the first boundary condition in (2), if

$$
J \rightarrow \min _{\varphi}
$$

Note the second boundary condition in (2) plays the role of transversality condition for the functional (4) and is satisfied 'automatically' for extremum solution. Note that Equation 3 is the Eulerian equation for the functional (4).

In what follows we will suppose that the material is locally orthotropic and the orientation of the axes of orthotropicity is unknown beforehand. Let us fix the unit vectors $\mathbf{e}_{1}^{0}, \mathbf{e}_{2}^{0}, \mathbf{e}_{3}^{0}$ of orthogonal coordinate system $x_{1}, x_{2}, x_{3}$ which is considered as a global system (see Figure 2). The principal directions unit vectors $\mathbf{e}_{1}, \mathbf{e}_{2}, \mathbf{e}_{3}$ of the heat conduction tensor $D$ of orthotropic material (axes of local symmetry) at the arbitrary point $\left(x_{1}, x_{2}, x_{3}\right) \in \Omega$ are related with the global coordinate vectors $\mathbf{e}_{1}^{0}, \mathbf{e}_{2}^{0}, \mathbf{e}_{3}^{0}$ by means of the rotation tensor $Q=Q(x)$ as

$$
\begin{aligned}
& \mathbf{e}_{i}=Q * \mathbf{e}_{i}^{0}=Q \times \mathbf{e}_{i}^{0} \quad(i=1,2,3) \\
& Q^{\mathrm{T}} \times Q=Q \times Q^{\mathrm{T}}=E
\end{aligned}
$$

where the symbol T means the operation of transposition and $E=\left\{\delta_{i j}\right\}$ - unit tensor, where $\delta_{i j}$ is a Kronecker symbol $(i, j=1,2,3)$ and $(*)$ is a tensor operation of the rotation. In the axes of symmetry of the orthotropic material, the heat conduction tensor $D$ is written as

$$
D=D_{i j} \mathbf{e}_{i} \otimes \mathbf{e}_{j}=D_{i j}^{0} Q \times \mathbf{e}_{i}^{0} \otimes Q \times \mathbf{e}_{j}^{0}=Q *\left(D_{i j}^{0} \mathbf{e}_{i}^{0} \otimes \mathbf{e}_{j}^{0}\right)=Q * D^{0}
$$

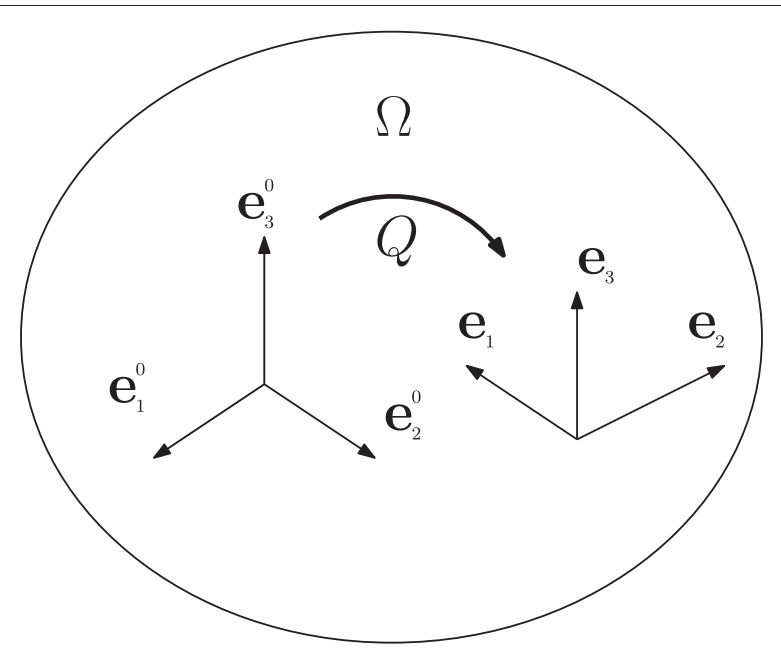

Figure 2 Transformation of global unit vectors to the local material principle vectors by rotation tensor $Q$. 
where $\otimes$ is the tensor product and

$$
D^{0}=D_{i j}^{0} \times \mathbf{e}_{i}^{0} \otimes \mathbf{e}_{j}^{0}
$$

The expression for the heat conduction tensor $D$ can be rewritten in the form

$$
D=D_{i j}^{0} Q \times \mathbf{e}_{i}^{0} \otimes Q \times \mathbf{e}_{j}^{0}=Q \times\left(D_{i j}^{0} \mathbf{e}_{i}^{0} \otimes \mathbf{e}_{j}^{0}\right) \times Q^{\mathrm{T}}=Q \times D^{0} \times Q^{\mathrm{T}}
$$

If $\varkappa_{i}$ and $\mathbf{e}_{i}^{0}$ i.e. eigenvalues and eigenvectors of the tensor $D^{0}$, i.e.

$$
D^{0} \times \mathbf{e}_{i}^{0}=\varkappa_{i}^{0} \mathbf{e}_{i}^{0}
$$

then $\varkappa_{i}^{0}$ and $\mathbf{e}_{i}=Q \times \mathbf{e}_{i}^{0}$ i.e. eigenvalues and eigenvectors of the tensor $D=Q * D^{0}$, i.e.

$$
D \times \mathbf{e}_{i}=\varkappa_{i}^{0} \mathbf{e}_{i}
$$

Taking into account Equations 7, 8 and 11, we will have Equation 12. In fact,

$$
D \times \mathbf{e}_{i}=\left(Q * D^{0}\right) \times\left(Q * \mathbf{e}_{i}^{0}\right)=Q *\left(D^{0} \times \mathbf{e}_{i}^{0}\right)=\varkappa_{i}^{0} Q * \mathbf{e}_{i}^{0}=\varkappa_{i}^{0} \mathbf{e}_{i}
$$

For given tensor $D^{0}$, the values of the functionals $J$ depend on the realization of $Q=$ $Q(x)$ and corresponding actual values of $\varphi=\varphi(x)$, minimizing the functional of energy dissipation (4) under constraints (2) (for considered $Q(x)$, i.e.

$$
J\left(Q, \varphi_{*}\right)=\min _{\varphi} J(Q, \varphi)
$$

\section{Uncertainties in orientation of orthotropic material and double-sided estimates}

If there is no data concerning material orientation, i.e. the tensor-function $Q=Q(x)$ $(x \in \Omega)$, characterizing material distribution is unknown, then it is very important to obtain the lower and upper bounds of $J$, i.e. to find the limit double-sided estimates $J_{\min }$ and $J_{\max }$, such that

$$
J_{\min } \leq J\left(Q, \varphi_{*}\right) \leq J_{\max }
$$

for any realization of $Q$ satisfying the condition (7).

To obtain reliable estimations of the dissipation energy functional $J$ and other important characteristics, we apply in the paper an approach based on the solution of two optimization problems. The following problem is devoted to finding the lower estimate

$$
J_{\min }=\min _{Q} J\left(Q, \varphi_{*}\right)=\min _{Q} \min _{\varphi} J(Q, \varphi)
$$

and another problem consists in the searching of the upper bounds

$$
J_{\max }=\max _{Q} J\left(Q, \varphi_{*}\right)=\max _{Q} \min _{\varphi} J(Q, \varphi)
$$

where min and max with respect to $Q$ in Equations 16 and 17 are determined under constraint (7). Operation min with respect to $\varphi$ in Equations 16 and 17 is performed taking into account boundary conditions from Equation 2.

In what follows, we will study the proposed approach and problems of searching the extremum of $J$ with respect to $Q$

$$
J \rightarrow \underset{Q}{\operatorname{extr}}
$$

and analyze extremum conditions and behavior equations. 


\section{Extremal conditions for orthotropic material orientation}

To derive extremum conditions, defining the orthogonal tensor of rotation $Q=Q(x)$ and characterizing the extremal orientations of orthotropy axes let us use the method of Lagrange multipliers and construct augmented functional

$$
\begin{aligned}
& J^{L}=J+J_{P} \\
& J_{P}=\int_{\Omega} P \cdots\left(Q^{\mathrm{T}} \times Q-E\right) \mathrm{d} \Omega \\
& J=\int_{\Omega} \nabla \varphi \times\left(Q * Q^{0}\right) \times \nabla \varphi \mathrm{d} \Omega=\int_{\Omega} \nabla \varphi \times\left(Q \cdot D^{0} \times Q^{\mathrm{T}}\right) \times \nabla \varphi \mathrm{d} \Omega
\end{aligned}
$$

where (..) between tensors mean double scalar product and symmetric tensor of second rank $P=P(x)(x \in \Omega)$ is Lagrange multiplier, specifying in $\Omega$ and corresponding to the condition of orthogonality (see Equation 7). The dissipation energy functional $J$ can be also rewritten as

$$
J=\int_{\Omega} B \cdots\left(Q \times D^{0} \times Q^{\mathrm{T}}\right) \mathrm{d} \Omega
$$

Here, by means of $B$, we denote the following symmetric second rank tensor

$$
B=\nabla \varphi \otimes \nabla \varphi, \quad B^{\mathrm{T}}=B
$$

and the symbol $\otimes$ is the tensor product.

Let us derive the following expressions for the first variations $\delta J$ and $\delta J_{P}$ with respect to variation $\delta Q$ of rotation tensor $Q$. We will have

$$
\delta J=\int_{\Omega} B \cdot\left(\delta Q \times D^{0} \times Q^{\mathrm{T}}+Q \times D^{0} \times \delta Q^{\mathrm{T}}\right) \mathrm{d} \Omega=2 \int_{\Omega} \delta Q \cdots\left(D^{0} \times Q^{\mathrm{T}} \times B\right) \mathrm{d} \Omega
$$

and

$$
\delta J_{P}=\int_{\Omega} P \cdots\left(\delta Q^{\mathrm{T}} \times Q+Q^{\mathrm{T}} \times \delta Q\right) \mathrm{d} \Omega=2 \int_{\Omega} \delta Q \cdots\left(P \times Q^{\mathrm{T}}\right) \mathrm{d} \Omega
$$

Taking into account the expressions (19) to (21), (24) and (25), we will find the expression for the total variation $\delta Q$ in the following form

$$
\delta J^{L}=\delta J+\delta J_{P}=2 \int_{\Omega} \delta Q \cdots\left(D^{0} \times Q^{\mathrm{T}} \times B+P \times Q^{\mathrm{T}}\right) \mathrm{d} \Omega
$$

Using the extremum condition

$$
\delta J^{L}=0
$$

and arbitrariness of $Q$, i.e. arbitrariness of $\delta Q$, we will have

$$
D^{0} \times Q^{\mathrm{T}} \times B+P \times Q^{\mathrm{T}}=0, \quad x \in \Omega
$$

Multiplying the relation (28) on $Q$ and using formulae (10) and (23) we find

$$
D \times \nabla \varphi \otimes \nabla \varphi=-Q \times P \times Q^{\mathrm{T}}, \quad x \in \Omega
$$

This relation means the symmetry of the second rank tensor

$$
(D \times \nabla \varphi) \otimes \nabla \varphi
$$

written in the left-hand side of the equality (29), i.e.

$$
(D \times \nabla \varphi) \otimes \nabla \varphi=\nabla \varphi \otimes(D \times \nabla \varphi)
$$


The equality (30) is satisfied if the vectors $D \times \nabla \varphi$ and $\nabla \varphi$ are parallel, i.e.

$$
D \times \nabla \varphi=\lambda \nabla \varphi
$$

where $\lambda$ is some scalar value.

\section{Double-sided estimates based on derived extremal conditions}

The necessary extremum condition (31) for dissipation energy functional $J$ with respect to rotation tensor $Q$, defining an extremal distribution of $Q$ and expressing the collinearity of the vectors $\nabla \varphi$ and

$$
D \times \nabla \varphi=\left(Q \times D \times Q^{\mathrm{T}}\right) \times \nabla \varphi
$$

is an eigenvalue problem. Consequently, the vector $\nabla \varphi$ is one of the eigenvectors of the heat conduction tensor $D$ :

$$
D \times \nabla \varphi=\lambda_{i} \nabla \varphi, \quad i=1,2,3
$$

Taking into account that the eigenvalues $\lambda_{i}$ of the tensors $D$ and $D^{0}$ are equal (see Equations 11 and 13) and given, we assume

$$
\lambda_{1}=\lambda_{\min }<\lambda_{2}<\lambda_{3}=\lambda_{\max }
$$

Substituting (32) into the Euler Equation (3) of the functional $J$, we obtain the equations that determine the stationary distribution of scalar function $\varphi=\varphi(x)$ :

$$
\nabla \times\left(\lambda_{i} \nabla \varphi\right)=0, \quad(i=1,2,3), \quad x \in \Omega
$$

in the case of specified rotation tensor $Q$ according to the equation

$$
\left(Q \times D^{0} \times Q^{\mathrm{T}}\right) \times \nabla \varphi=\lambda_{i} \nabla \varphi
$$

The elliptical partial differential Equation (34) with the boundary conditions

$$
(\varphi)_{\Gamma_{g}}=\varphi^{0}, \quad\left(\lambda_{i} \mathbf{n} \times \nabla \varphi\right)_{\Gamma_{i}}=0
$$

corresponding to conditions (2) with the relations (32) constitute the conventional boundary value problem describing, as it is well known, homogeneous or nonhomogeneous isotropic processes of the heat conductivity. Under some known additional constraints superimposed on the boundary shape $\Gamma=\Gamma_{g}+\Gamma_{i}$, where $\Gamma_{g} \cap \Gamma_{i}=0$, we have the existence and uniqueness of the solution of (34) and (36) with given $\lambda_{i}$.

If we assume that the same way of extremum orientation of the principle axes of orthotropy is realized for all domain $\Omega$, then $\lambda_{i}$ is constant in $\Omega$ and the considered heat conduction process is described by the classical boundary value problem

$$
\begin{aligned}
& \Delta \varphi=0, \quad x \in \Omega \\
& (\varphi)_{\Gamma_{g}}=\varphi^{0}, \quad(\mathbf{n} \times \varphi)_{\Gamma_{i}}=0
\end{aligned}
$$

for Laplace equation with mixed (in general case) boundary conditions. Here $\Delta$ is a Laplace operator acting in a three-dimensional space.

Note that the equality in Equation 37 means that in the case of the body with extremum orthotropy the heat conduction process is described by the same equation as in the isotropic case. If the domain $\Omega$ consists of several subdomains $\Omega_{i}$ such that

$$
\Omega=\cup \Omega_{i}, \quad \Omega_{i} \cap \Omega_{j}=0 \quad(i \neq j)
$$


and for each separate subdomain $\Omega_{i}$, the same extremum way of material orientation is taken, then the isotropic heat conduction process is realized for all considered subdomains.

Let us assume that the orthotropic material is distributed in accordance with the same extremum rule in the domain $\Omega$. Then we will have the 'isotropic' boundary value problem (37) and (38), and consequently the state variable $\varphi$ (inverse temperature) is independent of $\lambda_{i}$. As a result, we obtain the following minimal and maximal values of the considered quality functional $J$ :

$$
\begin{aligned}
& \min _{Q} J=\lambda_{\min } I \\
& \max _{Q} J=\lambda_{\max } I
\end{aligned}
$$

where

$$
I=\int_{\Omega}(\nabla \varphi)^{2} \mathrm{~d} \Omega
$$

Thus, the double-sided estimates of the energy dissipation functionals can be written as

$$
\lambda_{\min } \leq \frac{J}{I} \leq \lambda_{\max }
$$

\section{Two-dimensional case of extremal material orientation}

Separately, consider the two-dimensional case with plane domain $\Omega$. In this case

$$
\nabla \varphi=\left\{\frac{\partial \varphi}{\partial x_{1}}, \frac{\partial \varphi}{\partial x_{2}}\right\}, \quad x=\left\{x_{1}, x_{2}\right\} \in \Omega
$$

Then the element of orthogonal tensor $Q$ are represented in the form

$$
Q_{11}=Q_{22}=\cos \alpha \quad Q_{21}=-Q_{12}=\sin \alpha
$$

where $\alpha$ is the angle of rotation of the specified tensor $Q$. On the basis of Equation 35, we obtain an explicit expression relating the angle $\alpha=\alpha\left(x_{1}, x_{2}\right)$ with the function $\varphi=$ $\varphi\left(x_{1}, x_{2}\right)$. In Figure 3, the orientation of local orthotropicity is presented. For definiteness, assume that the vector $\nabla \varphi$, presented in Equation 44, correspond to the eigenvalue $\lambda_{i}$. Then the eigenvector $\mathbf{k}$, corresponding to the eigenvalue $\lambda_{j}(i \neq j)$ is

$$
\mathbf{k}=\left\{\frac{\partial \varphi}{\partial x_{2}},-\frac{\partial \varphi}{\partial x_{1}}\right\}
$$

which is orthogonal to the eigenvector $\nabla \varphi$ from Equation 44 . We form a scalar product of both sides of the vector equality (32) with the vector $\mathbf{k}$. We will have

$$
\mathbf{k} \times D \times \nabla \varphi=0
$$

This relation contains two separate cases. The first case

$$
\cos 2 \alpha=C, \quad \sin 2 \alpha=S
$$

where

$$
C=-\frac{\left(D_{11}^{0}-D_{22}^{0}\right)\left\{\left(\frac{\partial \varphi}{\partial x_{1}}\right)^{2}-\left(\frac{\partial \varphi}{\partial x_{2}}\right)^{2}\right\}+4 D_{12}^{0} \frac{\partial \varphi}{\partial x_{1}} \frac{\partial \varphi}{\partial x_{2}}}{(\nabla \varphi)^{2} \sqrt{\left(D_{11}^{0}-D_{22}^{0}\right)^{2}+4\left(D_{12}^{0}\right)^{2}}}
$$




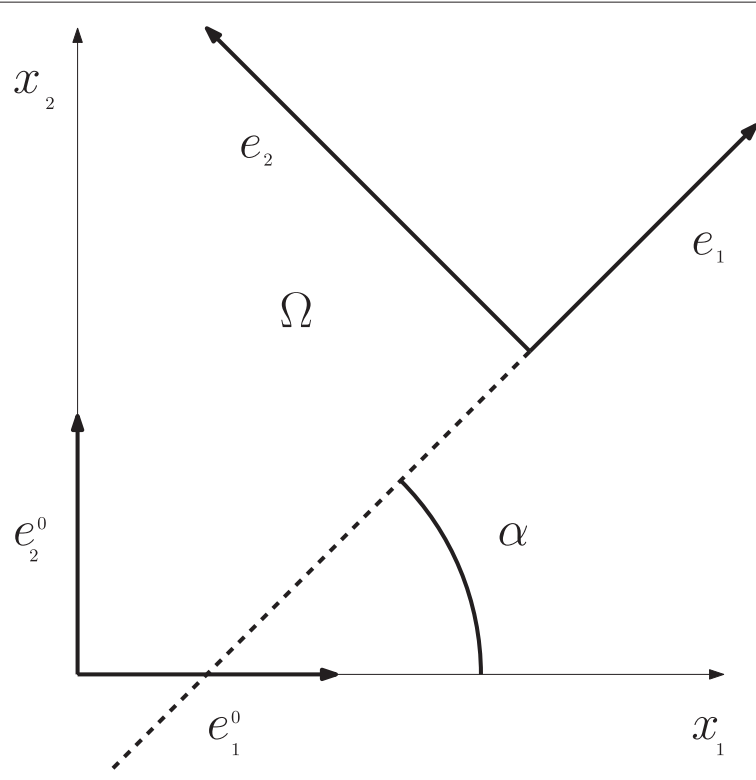

Figure 3 Orientation of local orthotropicity in a two-dimensional case.

and

$$
S=\frac{2\left(D_{11}^{0}-D_{22}^{0}\right) \frac{\partial \varphi}{\partial x_{1}} \frac{\partial \varphi}{\partial x_{2}}-2 D_{12}^{0}\left\{\left(\frac{\partial \varphi}{\partial x_{1}}\right)^{2}-\left(\frac{\partial \varphi}{\partial x_{2}}\right)^{2}\right\}}{(\nabla \varphi)^{2} \sqrt{\left(D_{11}^{0}-D_{22}^{0}\right)^{2}+4\left(D_{12}^{0}\right)^{2}}}
$$

corresponds to the smaller eigenvalue $\lambda_{1}\left(\lambda_{1}<\lambda_{2}\right)$. The second case

$$
\cos 2 \alpha=-C, \quad \sin 2 \alpha=-S
$$

corresponds to the larger eigenvalue $\lambda_{2}\left(\lambda_{2}>\lambda_{1}\right)$.

\section{Examples of double-sided estimates}

Suppose at first that the orthotropic material occupies the three-dimensional domain $\Omega$ situated between the internal sphere of radius $r_{1}$, where $r_{1}, r_{2}\left(r_{1}<r_{2}\right)$ is given values. The temperature of $\theta=\theta_{1}$ is defined at the internal boundary and the temperature $\theta=\theta_{2}$ is given at the external boundary, where $\theta_{1}<\theta_{2}$. Note that $\theta_{i},(i=1,2)$ are given and positive values. Thus, we consider the following boundary conditions:

$$
\begin{array}{ll}
\varphi=\varphi_{1}=\frac{1}{\theta_{1}}, \quad r=r_{1} \\
\varphi=\varphi_{2}=\frac{1}{\theta_{2}}, & r=r_{2}
\end{array}
$$

where $\varphi_{1}<\varphi_{2}$. Here, we use spherical coordinate system with the origin at $r=0$. From the properties of symmetry, it follows that the extremum orientations of the axes of orthotropy with

$$
\lambda_{1}=\lambda_{\min } \quad \text { and } \quad \lambda_{3}=\lambda_{\max }
$$

corresponding respectively to the cases

$$
J \rightarrow \min _{Q} \text { and } \quad J \rightarrow \max _{Q}
$$


are realized in radial direction. Besides, the gradient of $\varphi$, i.e. vector $\nabla \varphi$, and also the heat flux vector $\mathbf{q}$ are directed along the radius vector at each point of the domain $\Omega$. Note that the heat flux $\mathbf{q}$ is absent in circumferential directions. The following values characterize the extremal distribution of material:

$$
\begin{aligned}
& \mathbf{q}_{\min }=\lambda_{\min } N \mathbf{r}_{0}, \quad \mathbf{q}_{\max }=\lambda_{\max } N \mathbf{r}_{0} \\
& \lambda_{\min } I \leq J \leq \lambda_{\max } I \\
& N=\frac{\varphi_{2}-\varphi_{1}}{r_{2}-r_{1}}, \quad \mathbf{r}_{\mathbf{0}}=\frac{\mathbf{r}}{|\mathbf{r}|}
\end{aligned}
$$

where

$$
I=\int_{\Omega}(\nabla \varphi)^{2} \mathrm{~d} \Omega=\frac{4}{3} \pi N\left(\varphi_{2}-\varphi_{1}\right)\left(r_{1}^{2}+r_{1} r_{2}+r_{2}^{2}\right)
$$

and $\mathbf{r}_{\mathbf{0}}$ is a unit vector, oriented in radial direction.

Next let us consider the problem of finding the double-side estimates when a simply connected domain $\Omega$ occupied by the orthotropic material is a rectangular parallel-piped with the upper and lower faces at $x_{3}=-c$ and $x_{3}=c$ and side faces at $x_{1}= \pm a$ and $x_{2}= \pm b$. We use the Cartesian coordinate system $\left(x_{1}, x_{2}, x_{3}\right)$ and we assume that the temperature $\theta$ is given at the lower and upper faces and the side faces are thermally insulated, i.e. the boundary conditions have the form:

$$
\varphi=\varphi_{1}=\frac{1}{\theta_{1}}, \quad x_{3}=-c \quad \text { and } \quad \varphi=\varphi_{2}=\frac{1}{\theta_{2}}, \quad x_{3}=c
$$

and

$$
\mathbf{q} \times \mathbf{n}=\mathbf{n} \times D \times \nabla \varphi=0 \text { at } x_{1}= \pm a, x_{2}= \pm b
$$

where $\theta_{1}>0, \theta_{2}>0$ and $\left(\theta_{1}>\theta_{2}\right)$. Extremal material distribution and corresponding heat conduction processes are characterized by the existenec of level surfaces $x_{3}$ is constant $\left(-c<x_{3}<c \in \Omega\right.$ ) with a constant distribution of variable $\varphi$ (constant temperature $\theta$ ). The gradient of $\varphi$ is parallel to $x$-axis. Therefore the axes of orthotropy with minimal eigenvalue $\lambda=\lambda_{\min }$ (in the case $J \rightarrow \min _{Q}$ ) and with maximal eigenvalue $\lambda=\lambda_{\max }$ (in the case of $J \rightarrow \max _{Q}$ ) are oriented in a parallel way with respect to the axis $x_{3}$. Such orientation provides, respectively, either the minimium or the maximum of dissipation. For considered problem we will have

$$
\begin{aligned}
& \mathbf{q}_{\min }=\lambda_{\min } \Phi \mathbf{x}_{3}^{0}, \quad \mathbf{q}=\lambda_{\max } \Phi \mathbf{x}_{3}^{0} \\
& \min _{Q} J=\lambda_{\min } I, \quad \max _{Q} J=\lambda_{\max } I \\
& \Phi=\frac{\varphi_{2}-\varphi_{1}}{2 c}, \quad \nabla \varphi=\Phi \mathbf{x}_{3}^{0}, \quad \mathbf{x}_{3}^{0}=\frac{\mathbf{x}_{3}}{\left|\mathbf{x}_{3}\right|}
\end{aligned}
$$

where

$$
I=\int_{\Omega}(\nabla \varphi)^{2} \mathrm{~d} \Omega=\frac{2 a b}{c}\left(\varphi_{2}-\varphi_{1}\right)^{2}
$$

and $\mathbf{x}_{3}^{0}$ is an unit vector of the $x_{3}$-axis, obtained when the vector $\mathbf{x}_{3}$ is divided by its length $\left|\mathbf{x}_{3}\right|$.

\section{Conclusions}

In the case, when the coefficient $D_{i j}$ and the considered eigenvalues $\lambda_{i}$ are independent of $x=\left(x_{1}, x_{2}, x_{3}\right)$, then the anisotropic behaviour equation is reduced to the Laplace equation which describes the heat conduction of homogeneous isotropic body. Since the 
theory of the heat conduction of isotropic homogeneous solids is well developed and solution of the corresponding boundary value problem has been found (analytically and numerically) for most problems of practical importance, then this reduction allows to consider the above problem of obtaining of double-sided estimates to be solved.

Taking into account the conditions of uncertainties concerning material orientations, we obtain various estimations of the considered functionals and in particular limiting estimates known as double-sided or bilateral estimates. The search of double-sided estimates as it was shown is reduced to the solution of optimization problems and finding the extremal orientation of the orthotropy axes. The results can be applied for example to the optimization of the paper drying process, which has a significant role in energy consumption of the paper production.

\section{Acknowledgments}

The research was performed under financial support of RFBR (grant 11-08-00030-a), RAS Program 12, Program of Support of Leading Scientific Schools (grant 2611.2012.1) and Funding from Academy of Finland (grants no. 140221 and 269351).

\section{Author details}

${ }^{1}$ Institute for Problems in Mechanics, Russian Academy of Sciences, Pr. Vernadskogo, 101-1, Moscow 119562, Russia.

${ }^{2}$ Department of Mathematical Information Technology, University of Jyväskylä, Mattilanniemi 2, Agora, 40014 Jyväskylä, Finland.

Received: 16 April 2014 Accepted: 24 June 2014

Published: 21 August 2014

\section{References}

1. Banichuk, NV, Neittaanmäki, PJ: Structural Optimization with Uncertainties. Springer-Verlag, Dordrech (2010). ISBN 978-90-481-2517-3

2. Karlsson, M: On-line study and mathematical modeling of a paper dryer section. Åbo Akademi, Faculty of Chemical Engineering, Plant Design Laboratory (1984)

3. Lampinen, MJ, Toivonen, K: Application of a thermodynamic theory to determine capillary pressure and other fundamental material properties affecting the drying process. In: Drying '84, pp. 228-244. Hemisphere/McGraw-Hill, New York, (1984)

4. Lu, T, Shen, SQ: Numerical and experimental investigation of paper drying: heat and mass transfer with phase change in porous media. Appl. Thermal Eng. 27, 1248-1258 (2007)

5. Karlsson, M (ed.): Papermaking, Part 2 Drying, Volume 9 of Papermaking Science and Technology. Paperi ja Puu Oy (2009). ISBN 978-952-5216-37-0 (book 9).

6. Landau, D, Lifshitz, EM: Teoriya uprugosti (Theory of Elasticity, 2nd ed.). Pergamon, Oxford (1965)

7. Nowacki, W: Teoria sprezystosci. Pantwawe Wydawnictwo Naukowe, Warsaw (1970)

8. Berdichevsky, V: Variational principles of continuum mechanics. Springer, New York (2009)

doi:10.1186/s40467-014-0019-z

Cite this article as: Banichuk et al:: Reliable estimates in the anisotropic heat conduction problems. Journal of Uncertainty Analysis and Applications 2014 2:19.

\section{Submit your manuscript to a SpringerOpen ${ }^{\odot}$ journal and benefit from:}

- Convenient online submission

- Rigorous peer review

- Immediate publication on acceptance

- Open access: articles freely available online

- High visibility within the field

- Retaining the copyright to your article

Submit your next manuscript at $>$ springeropen.com 